\title{
GLACIAÇÃO SAMBURÁ (NEOPROTEROZÓICO-VENDIANO?) COMO POSSÍVEL AGENTE TRANSPORTADOR DE DIAMANTES NO ESTADO DE MINAS GERAIS
}

\author{
GUILHERME M. GONZAGA ${ }^{1}$
}

\begin{abstract}
RESUMO Este trabalho sugere que um segundo evento glacial (Neoproterozóico-Vendiano?) possa ter ocorrido no Estado de Minas Gerais como também em outros estados. É proposta a denominação de Glaciação Samburá para este evento. Este segundo evento glacial (Neoproterozóico) está muito bem representado em todo o mundo, porém não é uma glaciaçâo continental mas sim de altitude. No Brasil está diretamente relacionada aos processos tectônicos do Brasiliano (cerca de 600 m.a.). No Estado de Minas Gerais, evidências da Glaciação Samburá estão representadas, entre outros, pelos sedimentos glaciogênicos da Formação Samburá (Grupo Bambuí), na região da Serra da Canastra, Lagoa Formosa, Tiros, Quintinos, etc. Também podem estar representados pelos diamictitos (e outros sedimentos) da base do Grupo Vazante na região de Coromandel (Faixa Brasília) e por sedimentos glaciogênicos do topo da Bacia Andrelândia na Faixa Ribeira. É possível, e bastante provável na opinião do autor, que os processos glaciais (Glaciação Samburá) tenham atuado como agentes no transporte de diamantes em algumas regiões do Estado de Minas Gerais.
\end{abstract}

Palavras-chave: Glaciação; Vendiano; Agente Transportador; Diamante

\begin{abstract}
THE SAMBURÁ GLACIATION (NEOPROTEROZOIC - VENDIAN?) AS A POSSIBLE TRANSPORTING AGENT FOR DIAMONDS IN THE MINAS GERAIS STATE This work suggests that a second Neoproterozoic glacial event that possibly happened during the Vendian in some regions of Minas Gerais and in some other states of Brazil be called Samburá Glaciation. This second Neoproterozoic glaciation is very well represented in the world. It did not occur on a continental scale like the first one called Jequitai Glaciation in Brazil, but comprises in an altitude glacial event. In Brazil, this event relates to the elevations created by the Brasiliano Tectonic Event (ca. $600 \mathrm{~m} . \mathrm{a})$. In the Minas Gerais State, evidence of the Samburá Glacial process is represented by glaciogenic sediments from the Samburá Formation-Bambui Group in the regions of Serra da Canastra, Lagoa Formosa, Tiros, Quintinos, etc. It could also be related to diamictites from the base of Vazante Group in the Coromandel region (Brasilia Belt), as well as to sediments from the top of the Andrelândia Basin in the Ribeira Belt. It is possible and, in the author's view, highly probable that the glacial process (Samburá Glaciation) acted as a diamond transportation agents in some parts of the Minas Gerais State.
\end{abstract}

Keywords: Glaciation; Vendian; Transport Agent; Diamond

INTRODUÇÃO Barbosa et al. (1970) citaram a ocorrência de conglomerados intraformacionais (Fácies Molássica Samburá) na estrada entre Presidente Olegário e Lagamar e nas proximidades de Campos Altos. Estes sedimentos foram posicionados, pelos autores, sobre a Formação Sete Lagoas (Grupo Bambuí) e correlacionados com os "Conglomerados" Samburá (Branco 1956) na região da Serra da Canastra (São
Roque de Minas).

Dardenne et al. (1978), Gonzaga \& Tompkins (1991) e outros consideraram estes sedimentos como glaciogênicos e relacionados com o evento glacial Jequitaí (Glaciação Jequitaí de Couto \& Bez 1981), que apresenta um registro de natureza regional na base do Grupo Bambuí (Dardenne et al. 1978). Esta hipótese está descartada devido ao nítido posicionamento

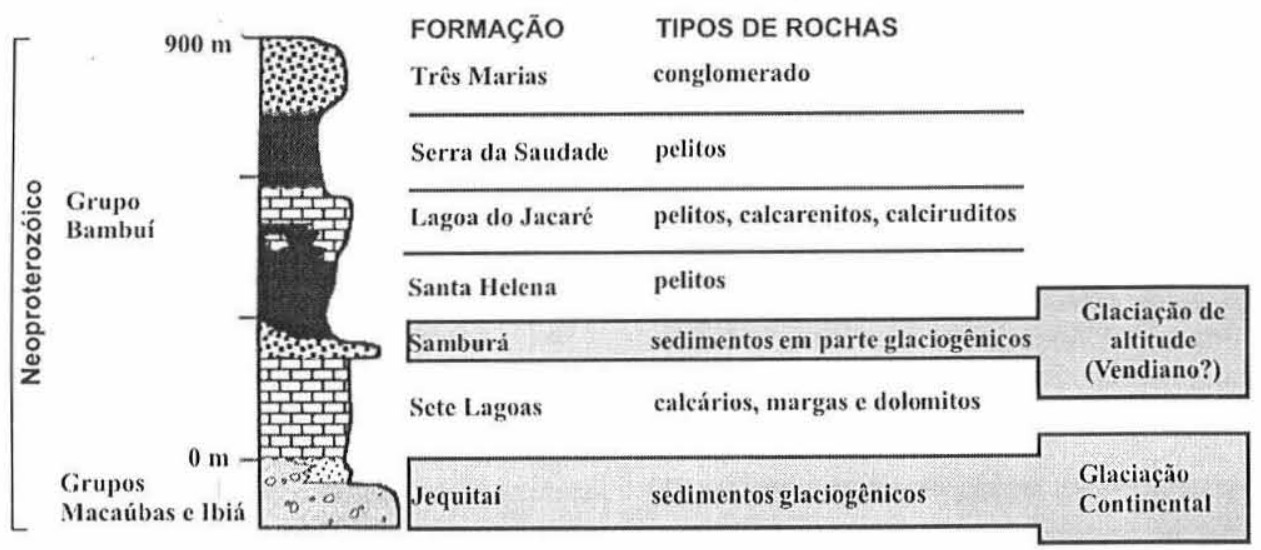

Figura I - Posicionamento das glaciações Jequitaí e Samburá em relação ao Grupo Bambuí (adaptada de Alkmim et al. 2001). 
dos "Conglomerados Samburá" sobre os sedimentos carbonáticos da Formação Sete Lagoas do Grupo Bambuí, conforme Castro \& Dardenne (1996) e outros. Alkmin et al. (2001) consideraram os sedimentos Samburá como uma formação do Grupo Bambuí, posicionada sobre a Formação Sete Lagoas e sotoposta à Formação Santa Helena (Fig.1).

A Formação Samburá (Alkmin et al. 2001) em São Roque de Minas, região da Serra da Canastra, apresenta segundo Gonzaga \& Tompkins (1991) conglomerados polimíticos, diamictitos e sedimentos pelíticos com seixos pingados (drop stones). A matriz dos diamictitos e também alguns níveis pelíticos freqüentemente contêm fosfatos $\left(\mathrm{P}_{2} \mathrm{O}_{5}\right)$ que podem atingir teores de $20 \%$ em superfície (lateritas) e teores de $5 \%$ na matriz dos diamictitos e nos sedimentos pelíticos.

Os diamictitos apresentam clastos (de grânulos a matacões) dispersos em uma matriz síltica-argilosa, algumas vezes arenosa. Os clastos são principalmente de quartzito e quartzo, ocorrendo também gnaisse, filito, carbonato e chert vermelho. Blocos e matacões pentagonais (flat-iron) são freqüentes (Gonzaga \& Tompkins 1991). Os sedimentos da Formação Samburá (Grupo Bambuí) foram apontados, na região da Serra da Canastra, como glaciogênicos e considerados possivelmente diamantíferos (Branco 1956, Gonzaga \& Tompkins 1991).

Seer et al. (1987) identificaram e descreveram diamictitos com matriz argilosa a síltica, que gradam para ardósias, associados ao Grupo Bambuí na região de Lagoa Formosa-MG. Os clastos presentes nos diamictitos (15 a $20 \mathrm{~cm}$ podendo atingir Imetro) são subangulares a subarredondados e constituídos principalmente por metassiltito e meta-arenito e ocasionalmente por ardósia e arcósio. Gnaisse, granito, metaconglomerado e jaspelito são raros. Seer et al. (1987) também identificaram jaspelitos em contato concordante com ritmitos. Os jaspelitos apresentam lâminas de chert e hematita intercaladas com lâminas de grãos detríticos ("BIF"). No Rio São Bento, região de ocorrência da seqüência metassedimentar de Seer et al. (1987), foi encontrado um diamante com 164 quilates que, na opinião de Gonzaga \& Tompkins (1991), poderia estar relacionado com os diamictitos (Formação Samburá).

Os sedimentos da Formação Samburá (Grupo Bambuí), que em parte são glaciogênicos, possivelmente representam um evento glacial ocorrido, em todo o mundo, no final do Neoproterozóico (Vendiano). Este evento está bem definido no Brasil apenas na região a oeste de Cuiabá (Mato Grosso), no Gráben de Corumbá, e na Serra da Bodoquena, no Mato Grosso do Sul (Alvarenga et al. 2000).

No decorrer deste trabalho será demonstrado que a Glaciação Samburá no Estado de Minas Gerais, como possivelmente também em outros estados, atuou como um agente transportador de diamantes. Várias ocorrências diamantíferas consideradas enigmáticas poderão ter suas origens elucidadas através dos processos da Glaciação Samburá

\section{GLACIAÇÃO VENDIANA (NEOPROTEROZÓICO)} Segundo Evans (2000), no mínimo dois grandes eventos glaciais, de natureza global, ocorreram no Neoproterozóico. O primeiro, a cerca de 740 milhões de anos (Sturtiano) e o segundo, a cerca de 600 milhões de anos (Vendiano). Estes dois eventos glaciais estão muito bem caracterizados na porção sul do continente africano. Segundo Frimmel \& Fölling (2000) datações (U- Pb em zircão, $\mathrm{Pb}-\mathrm{Pb}$ em carbonato) e estudos isotópicos $\left({ }^{13} \mathrm{C},{ }^{87} \mathrm{~S} /{ }^{86} \mathrm{~S}\right)$ permitem uma correlação com épocas glaciais do Sturtiano e Vendiano. No Brasil também são apontados dois eventos glaciais durante o Neoproterozóico (Alvarenga 1990, Alvarenga \& Trompette 1992).

A Glaciação Vendiana, na Namíbia, apresenta claros registros no Grupo Witvlei (650-530 milhões de anos) que é constituído, entre outros tipos litológicos, por diamictitos com clastos dispersos em uma matriz arenosa fina a pelítica. Os clastos podem atingir mais de $50 \mathrm{~cm}$ e apresentam-se freqüentemente facetados e estriados (Hegenberger 1993). O Grupo Witvlei pode ser correlacionado na África do Sul com a Formação Numees que, na opinião de Van Wyk \& Pienaar (1986) e Rouffaer (1988), é uma formação glaciogênica e possivelmente portadora de diamantes. Eerola (1995) correlaciona os sedimentos glaciogênicos do Vendiano da Namíbia/África do Sul com diamictitos e outros sedimentos que ocorrem no Supergrupo Camaquã, na região de Lavras do Sul, Estado do Rio Grande do Sul. Nestes sedimentos foram identificados fósseis da fauna de Ediacara, o que comprova a idade vendiana (Rosa 2000). No Uruguai ocorre o Grupo Arroyo Del Soldado que, além de apresentar fósseis do Vendiano, inclui em suas litologias uma formação ferrífera bandeada ("BIF") que favorece a hipótese de um evento glacial ocorrido no Vendiano (Basei et al. 2000). Há evidências de pelo menos dois episódios de clima frio no Grupo Arroyo Del Soldado (Gaucher \& Sprechmann 2000).

A oeste de Cuiabá (Mato Grosso), na borda do Cráton Amazônico, ocorre o Cinturão Paraguai, relacionado ao evento Brasiliano (cerca de 600 milhões de anos). Na base da seqüência ocorrem sedimentos glaciogênicos e turbidíticos pertencentes à Formação Puga e ao Grupo Cuiabá (Alvarenga \& Trompette 1992). Na opinião de Gonzaga \& Tompkins (1991), é provável que os diamictitos da Formação Puga sejam responsáveis, em grande parte, pela distribuição de diamantes na região. Ocorre uma associação espacial entre as fácies glaciogênicas grosseiras (tilitos) e as ocorrências diamantí-feras. $\mathrm{O}$ autor do presente trabalho teve a oportunidade de estudar, no mínimo, seis ocorrências diamantíferas diretamente relacionadas aos tilitos. Na região não ocorrem possíveis fontes diamantíferas primárias (kimberlitos e/ou lamproitos). Almeida (1968) acredita que uma quantidade apreciável de diamantes tenha sido obtida a partir dos sedimentos arenosos do Grupo Alto Paraguai (topo do Cinturão Paraguai) sem que se saiba ainda qual seja sua origem. Os sedimentos do Grupo Alto Paraguai possivelmente incorporaram diamantes a partir do retrabalhamento dos sedimentos glaciogênicos da base.

Em pelitos intercalados com carbonato que ocorrem $400 \mathrm{~km}$ a SSW de Cuiabá, sobrepostos aos sedimentos glaciogênicos e sotopostos ao Grupo Alto Paraguai, ocorrem fósseis (Fauna de Ediacara) de idade Vendiana (Fairchild 1978, Walde et al. 1982). Também é importante ressaltar que Alvarenga et al. (2000) claramente associam uma formação ferrífera (BIF) com os sedimentos, em parte glaciogênicos, dos Grupos Jacadigo (Mato Grosso do Sul) e Boqui (Bolívia) que são correlacionados à Formação Puga e ao Grupo Cuiabá.

A questão da origem da Glaciação Vendiana é um assunto polêmico em todo o mundo. Inúmeras opiniões divergentes podem ser encontradas na literatura geológica internacional. Um ponto que apresenta grande convergência de opiniões é a relação entre as glaciações e processos tectônicos. Os dados disponíveis não indicam uma natureza continental em larga escala para a Glaciação Vendiana. Ela é eminentemente de altitude, não podendo ser comparada com a primeira glaciação neoproterozóica no Brasil (Glaciação Jequitaí) que apresenta 
uma natureza continental (Karfunkel et al. 2000). Todavia isso não significa que localmente a Glaciação Jequitaí não tenha tido uma influência da altitude. Pedrosa-Soares et al. (2000), através do estudo de zircões (SHRIMP) do Grupo Macaúbas em Minas Gerais (Glaciação Jequitaí), obteve valores da ordem de $983 \pm 10$ Ma para a população mais jovem. Este valor encontrado indica uma idade máxima para esta primeira glaciação neoproterozóica.

NOVOS DADOS DE CAMPO Recentemente o autor, juntamente com o geólogo F. M. Rego, visitou a região de Carmo do Paranaíba, Lagoa Formosa, Quintinos, Tiros e o Vale do Rio da Prata, no Estado de Minas Gerais. Na região do Rio São Bento (Lagoa Formosa) observou a seqüência metassedimentar de Seer et al. (1987) e concluiu que os diamictitos são glaciogênicos. Ocorrem clastos pentagonais ("flat-iron") nos diamictitos e seixos pingados ("dropstones") em sedimentos pelíticos. Na Fazenda do Vandinho (UTM: 0367525/7906932) exatamente sobre os diamictitos foi encontrado um diamante de 164 quilates (Estado de Minas -20 de março de 1986). Um outro grande diamante (324 quilates) também foi encontrado no Rio São Bento (Reis 1959). Na região da ponte sobre o Rio Abaeté, na estrada entre Quintinos e Tiros (UTM: 03933316/790672) ocorrem garimpos de diamantes, tanto à montante quanto à jusante. Os garimpos estão localizados sobre diamictitos esverdeados que contém clastos, principalmente de metassiltitos e meta-arenito (Formação Samburá). Nesta região do rio Abaeté, grandes diamantes também foram encontrados. Barbosa et al. (1970) citam pedras de 350, 328, 238 e 215 quilates.

Próximo ao povoado de Porto Diamante, na margem do Rio da Prata (UTM: 0362185/8029960), também ocorrem grandes diamantes. O famoso diamante "007", com cerca de 400 quilates, foi encontrado nesta região. Associados aos garimpos ocorrem blocos e matacões de quartzito, sendo que alguns deles apresentam formas pentagonais (flat-iron) e possíveis estrias. O maior matacão observado $(4 \times 2,5 \times 2 \mathrm{~m})$ de forma alguma poderia ter sido transportado para o Vale do Rio da Prata a não ser através de processos glaciais. Os afloramentos de quartzitos mais próximos encontram-se a dezenas de quilômetros a oeste.

Nesta região de Minas Gerais ocorre o Grupo Areado (Cretáceo Inferior) que na sua formação basal (Abaeté), apresenta um conglomerado diamantífero (Hasui \& Penalva 1970, Gonzaga \& Tompkins 1991) com clastos de quartzito. Freyberg (1932 in: Guimarães 1951) sugeriu uma proveniência longínqua de oeste para os clastos de quatzito. Este geólogo pioneiro (Freyberg) achou necessária a atuação de processos glaciais para justificar o transporte, a longa distância, dos clastos de quartzitos. Tal fato levou Freyberg a correlacionar os sedimentos do Grupo Areado com os sedimentos glaciogênicos (permocarboníferos) da Bacia do Paraná. Obviamente a correlação foi incorreta, porém, a observação da necessidade do envolvimento de processos glaciais no transporte dos clastos de quartzito, a longa distância, foi brilhante. Campos (1996) aponta os processos de debris-flow como os mais importantes na sedimentação da Formação Abaeté nesta região do Estado de Minas Gerais. Este tipo de sedimentação não se distancia a mais de $10 \mathrm{~km}$ das áreas soerguidas (Campos 1996). Os processos fluviais não são mecanismos capazes de transportar clastos e/ou diamantes a dezenas/ centenas de quilômetros, mas o transporte à longa distância é compatível com os processos glaciais (Campos \&
Gonzaga 1999). Uma revisão dos processos glaciais atuantes no transporte e concentração de diamantes foi apresentada por Gonzaga \& Campos (2001). É bastante provável que os clastos de quartzito foram transportados, a partir de oeste, através de processos glaciais durante a Glaciação Samburá. Os sedimentos, em parte glaciogênicos, da Formação Samburá (Grupo Bambuí) foram retrabalhados por processos de debris-flow que atuaram na deposição da Formação Abaeté (Grupo Areado), no Cretáceo Inferior.

Os diamantes que ocorrem associados aos sedimentos da Formação Samburá são, na sua imensa maioria, provenientes do retrabalhamento do Grupo Ibiá (Neoproterozóico) a oeste. Os sedimentos do Grupo Ibiá (em parte glaciogênicos) são correlacionados aos sedimentos Jequitaí/Macaúbas (Dardenne et al. 1978).

A Glaciação Jequitaí, responsável pela deposição do Grupo Ibiá, provavelmente transportou diamantes a partir de fontes primárias localizadas no Cráton do São Francisco. Existe uma nítida associação espacial entre ocorrências diamantíferas e os sedimentos do Grupo Ibiá (Gonzaga \& Tompkins 1991, Gonzaga et al. 1994).

A formação basal do Grupo Ibiá (Fm Cubatão) apresenta diamictitos com matriz pelítica-carbonatada na qual flutuam clastos de diferentes tamanhos, formas e litologias. Os clastos chegam a atingir mais de $1 \mathrm{~m}$ (matacões). Alguns clastos são pentagonais e alguns seixos de quartzito são estriados. Os clastos predominantes são de quartzo e quartzito, mas também ocorrem rochas graníticas, básicas, filitos, calcários, chert e formação ferrífera (Pereira 1992).

Para a região do Triângulo Mineiro, Gonzaga \& Tompkins (1991) apresentaram um complexo esquema de reciclagem sedimentar envolvendo fontes primárias pré-cambrianas (Cráton do São Francisco), metaconglomerados do Mesoproterozóico, sedimentos da Glaciação Jequitaí, sedimentos glaciais do Permo-Carbonífero, sedimentos do Cretáceo (inferior e superior) e sedimentos cenozóicos. Também houve uma participação insignificante de fontes diamantíferas primárias da região, durante o Cretáceo. Esta complexa reciclagem sedimentar foi responsável pela presença da imensa maioria dos diamantes nesta região da Faixa Brasília. Contudo o esquema da reciclagem sedimentar encontra-se incompleto. Está faltando inserir a participação dos processos da Glaciação Samburá.

A possível, e bastante provável, presença de diamantes em sedimentos relacionados à Glaciação Jequitaí na Faixa Brasília (Grupo Ibiá) é reforçada pela presença de diamantes, em sedimentos da mesma glaciação, sobre o Cráton do São Francisco e na Faixa Araçuaí (Gonzaga \& Dardenne 1991, Campos \& Gonzaga 1999, Dardenne \& Schobbenhaus 2001, Karfunkel et al.2001). A presença de diamantes em sedimentos glaciogênicos da Glaciação Jequitaí foi apontada, inicialmente, por geólogos pioneiros como Derby (1906), Moares \& Guimarães (1930) e Guimarães (1951).

GLACIAÇÃO SAMBURÁ Castro \& Dardenne (1996) estudando o "Conglomerado Samburá" na região da Serra do Pimenta (SW de Minas Gerais) também apontaram uma proveniência a partir de oeste. Além disso, associaram estes sedimentos com uma bacia do tipo foreland. Um ambiente de sedimentação fan-delta foi sugerido para o "Conglomerado Samburá". O presente trabalho não é contrário às observações básicas de Castro \& Dardenne (1996), no entanto acrescenta uma glaciação de altitude (Glaciação Samburá) aos sistemas de 
fan-delta.

As informações até aqui apresentadas indicam que é bastante provável a existência de registros da Glaciação Samburá (Vendiano?) no Estado de Minas Gerais. Ultimamente novas informações da literatura geológica têm trazido uma importante contribuição a favor desta glaciação.

Fernandes et al. (2000) descrevem uma seqüência metassedimentar neoproterozóica próxima a Fortaleza de Minas e Itaú de Minas que compreende mármores, clorita e sericita filitos, quartzitos, além de rochas fosfatadas e formação ferrífera $(B I F)$. Segundo aqueles autores, a presença da formação ferrífera é indicativa de um suprimento pobre em terrígenos durante um evento glacial e da presença de águas com bastante oxigênio que promoveram uma oxidação e precipitação de ferro. Os fosfatos (marinhos) seriam formados pela mudança das condições glaciais para não glaciais através da redução da solubilidade dos fosfatos em águas quentes. $\mathrm{O}$ teor de $\mathrm{P}_{2} \mathrm{O}_{5}$ nos metassedimentos chega a atingir 25\% (Carvalho et al. 2000). Esta sequiência metassedimentar no extremo sul da Faixa Brasília, provavelmente é correlacionável com a seqüência metassedimentar de Seer et al. (1987) na região de Lagoa Formosa (Formação Samburá).

Gonzaga \& Tompkins (1991) apontaram a ocorrência de diamictitos com matriz fosfatada na região da cabeceira do Rio Santo Antônio do Bonito, no Município de Coromandel (Faixa Brasília). Nesta região foram encontrados os diamantes Presidente Vargas (726,6 quilates) e Darcy Vargas (460 quilates). Segundo Gonzaga \& Tompkins (1991), o diamante Presidente Vargas foi descoberto na Fazenda do Rufino, exatamente sobre afloramentos de diamictito com matriz fosfatada, pertencente ao Grupo Ibiá. Posteriormente este diamictito foi posicionado por Souza (1997) e Dardenne et al. (1997) na base do Grupo Vazante (Fm. Retiro). No Rio Santo Antônio do Bonito, os diamictitos da Formação Retiro (Grupo Vazante) apresentam seixos, blocos e matacões de granitóides, metassiltitos, ardósias, quartzitos, dolomitos e calcários dispersos em uma matriz argilosa ou argilo-carbonatada (Souza 1997). Dardenne (2000) descreve o Grupo Vazante (Neoproterozóico) como constituído, na base, pelas formações Retiro (quartzito, fosforita, diamictitos e ardósias) e Rocinha (ritimitos e ardósias fosfatadas). A seguir ocorre um conglomerado (Formação Arrependido) e uma vasta seqüência carbonática com algumas intercalações pelíticas (Formações Lagamar, Serra do Garrote, Serra do Poço Verde, Morro do Calcário e Serra da Lapa).

Dardenne (2000) indica uma proveniência a partir de oeste para a base do Grupo Vazante (Fig.2), o que sugere retrabalhamento do Grupo Ibiá. Os diamantes hoje presentes na região de Coromandel seriam, na sua imensa maioria, provenientes do Grupo Ibiá (Gonzaga \& Tompkins, 1991) e dos diamictitos da base do Grupo Vazante, segundo opinião do autor do presente trabalho. Informações básicas a respeito do porquê a região de Coromandel (Faixa Brasília) não é propícia à presença de fontes primárias diamantíferas podem ser encontradas no trabalho de Gonzaga et al. (1994).

É provável que a base do Grupo Vazante (formações Retiro e Rocinha) seja correlacionada com a Formação Samburá (Grupo Bambuí). Dardenne \& Schobeenhaus (2000) admitem um ambiente deposicional glacio-marinho para os fosfatos da base do Grupo Vazante. Azmy et al. (2000) apontam valores entre 0.706 e $0.7075\left({ }^{87} S_{r} /{ }^{86} S_{r}\right)$ para dolomitos do Grupo Vazante, valores que são comparáveis com a assinatura isotópica $\left(\mathrm{S}_{\mathrm{r}}\right)$ dos mares do Vendiano.
Segundo Dardenne et al. (1998), o Grupo Vazante foi depositado em uma bacia afetada por rápida subsidência instalada nos primeiros fronts de cavalgamento da Faixa Brasília, sendo portanto uma bacia de foreland.

Um contexto glaciogênico é compatível com a base do Grupo Vazante (Fig.2). A Glaciação Samburá teria sido favorecida por uma diferenciação tectônica criando áreas positivas (blocos com soerguimento) e negativas (bacias de subsidência) que proporcionariam condições favoráveis ao desenvolvimento de glaciação de altitude. Este conceito foi detalhado por Schermerhorn (1983).

Ainda referente ao Estado de Minas Gerais é conveniente citar os trabalhos de Leonardos (1940) e Ebert (1958). O primeiro autor descreveu a ocorrência do "tilito metamórfico de Carandaî", e o segundo propôs uma estratigrafia para a região de Carandaí.

O "tilito metamórfico de Carandaí", segundo Leonardos (1940) é composto por clastos de vários tipos de gnaisses. Com uma menor freqüência ocorrem granitos e calcários. Os clastos variam desde grânulos até matacões com mais de $1 \mathrm{~m}^{3}$. Leonardos (1940) sugeriu uma correlação com os sedimentos glaciogênicos Jequitaí/Macaúbas (Glaciação Jequitaí), opinião reforçada por Karfunkel \& Noce (1983), Karfunkel et al. (1983), Hoppe et al. (1984).

Ebert (1958) propôs para a região três formações (da base para o topo): Formação Carandaí, englobando o "tilito metamórfico" de Leonardos (1940). Formação Barroso, constituída por rochas clásticas-carbonáticas (atualmente cálcio-filitos) com grandes lentes de calcário com níveis argilosos, aqui considerada como equivalente à Formação Sete Lagoas, do Grupo Bambuí. Formação Prados. Segundo Ebert (1958) "As observações feitas na Formação Prados permitem também certas conclusões sobre as condições de sua origem. O elevado teor $\mathrm{em}$ plagioclásio num sedimento de granulação tão fina seria inexplicável num clima quente, em que o plagioclásio é um dos primeiros minerais a se decompor; o clima deve ter sido, pois, muito moderado, até frio. O ritmo regular da sedimentação, semelhante ao de um varvito sustenta esta conclusão..." Os sedimentos "várvicos" da Formação Prados talvez possam estar relacionados com o evento Glacial Samburá. Trouw et al. (2000) citam a presença de biotita filito (pelito feldspático) no topo da seqüência da Bacia Andrelândia (Neoproterozóico),

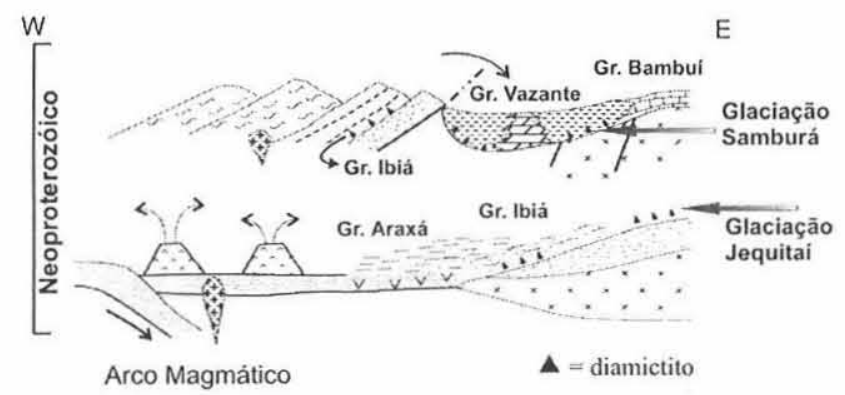

Figura 2 - Modelo esquemático mostrando a natureza continental da Glaciação Jequitai e a natureza de altitude da Glaciação Samburá (adaptada de Dardenne 2000). 
relacionados a uma mudança climática envolvendo condições glaciais. Também ocorrem diamictitos e seqüências pelíticas com "dropstones" (Trouw et al. 2000).

É, no mínimo, uma grande coincidência o fato da ocorrência de diamante $\mathrm{n}^{\circ} 361$ do Mapa Geológico do Brasil na escala 1:2.500.000 (Schobbenhaus et al. 1981) situada a SW de Andrelândia, estar localizada sobre metassedimentos da Bacia Andrelândia. Esta ocorrência, na Faixa Ribeira, pode estar relacionada com o evento Glacial Samburá.

Kaufman (1998), levando em consideração vários trabalhos anteriores, sugeriu que os carbonatos da Formação Sete Lagoas (Grupo Bambuí) apresentam na base valores negativos de ä ${ }^{13} \mathrm{C}$ (Influência da Glaciação Jequitaí) que tornam-se positivos em direção ao topo e novamente voltam a apresentar valores negativos. A inversão (no topo da Formação Sete Lagoas) de valores positivos para negativos pode indicar um segundo evento glacial não documentado (Kaufman, 1998). Este segundo evento glacial Neoproterozóico seria a Glaciação Samburá.

A POSSÍVEL PRESENÇA DA "GLACIAÇÃO SAMBURÁ” EM OUTROS ESTADOS Os sedimentos glaciogênicos que ocorrem no Domo de Cristalina em Goiás estão correlacionados com os sedimentos glaciogênicos Jequitaíl Macaúbas (Dardenne et al. 1978, Faria 1985, Cukrov 1999). Na opinião de Cukrov (1999) estes sedimentos foram depositados em um ambiente glacio-marinho. Trompette (1994) admite um posicionamento diferente para esses sedimentos $\mathrm{e}$ aponta uma similaridade com os diamictitos descritos por Guimarães et al. (1986) na região de Formosa-GO e com a seqüência metassedimentar de Seer et al. (1987) em Minas Gerais (Lagoa Formosa). Talvez os sedimentos glaciogênicos de Cristalina e Formosa, no Estado de Goiás, sejam produtos de processos glaciais ocorridos durante a "Glaciação Samburá”. Uma correlação do diamictito Samburá em Minas Gerais com o diamictito de Formosa-GO, já foi sugerida por Gonzaga \& Tompkins (1991), através da presença de fosfato tanto nos diamictitos Samburá (Gonzaga \& Tompkins 1991) como nos diamictitos de Formosa (Dardenne et al. 1978).

Gonzaga \& Tompkins (1991) admitem que os diamictitos que ocorrem no Domo de Cristalina-GO sejam diamantíferos. Na opinião dos referidos autores, no Rio São Bartolomeu, afluente do Rio Corumbá (Imediações do Domo de Cristalina) freqüentemente diamantes são recuperados, o mesmo ocorrendo em pequenas drenagens do Município de Cristalina.

$\mathrm{Na}$ Bahia, a presença de diamantes em conglomerados da Formação Salobro (Grupo Rio Pardo), na região de Canavieiras foi comprovada por O. Derby (Baptista et al. 1984). Segundo Leonardos (1940), D. Guimarães sugeriu uma origem glacial para os conglomerados da Formação Salobro (Neoproterozóico) na Bacia do Rio Pardo. Rocha-Campos \& Hasui (1981) indicam eventualmente a presença de diamictitos associados à Formação Salobro. Talvez os conglomerados flúvioglaciais e os diamictitos da Formação Salobro sejam produtos de processos glaciais da "Glaciação Samburá". Caso esta hipótese seja verdadeira, é possível que a Glaciação Jequitaí esteja representada, no Grupo Rio Pardo, pelos sedimentos da Formação Panelinha, sua unidade basal. Esta idéia já foi anteriormente apresentada por Silva Filho (1974). É oportuno reforçar que os conglomerados flúvio-glaciais da Formação Salobro são indiscutivelmente diamantíferos (Gonzaga \& Tompkins 1991), sendo um claro exemplo da presença de diamantes em sedimentos glaciogênicos no Brasil.
Caputo (1984) aponta, no embasamento da borda oeste da Bacia do Parnaíba (Cinturão Araguaia), a ocorrência de diamictitos e itabiritos associados a filitos e quartzitos (Grupo Tocantins). Esta sequiência apresenta uma conexão com a "Rokel Series" no noroeste africano que compreende tilitos, filitos com intercalações de calcário e quartzito, itabirito e rochas metavulcânicas (Torquato \& Cordani 1981). Dados de subsuperfície da Petrobrás, em poços da Bacia de São Luís no Estado do Maranhão, indicam a existência de diamictitos de origem possivelmente glacial associados a metapelitos. Os diamictitos estariam relacionados à fase final do Brasiliano (Caputo 1984). Os diamictitos acima referidos possivelmente são correlacionáveis tanto à Formação Puga em Mato Grosso (Cinturão Paraguai) quanto à Rokel Series no oeste africano, sendo portanto registro da segunda glaciação neoproterozóica, que tanto no Cinturão Paraguai (Alvarenga 1990, Alvarenga \& Trompette 1992) quanto na "Rokel Series" (Culver \& Williams 1978, Villeneuve 1987, Trompette 1994 e outros) é do Vendiano.

Segundo Pimentel et al. (1999), a colisão que resultou na porção sul da Faixa Brasília iniciou há cerca de 750 Ma.. Já os cavalgamentos em direção a leste ocorreram entre 640 a 620 Ma. (Valeriano 1999). Alkmin et al. (2000) mostram desenvolvimento escalonado para diferentes entidades do Brasiliano. Segundo estes autores, o último cinturão (ou faixa) a se desenvolver foi a Faixa Paraguai por volta de $550 \mathrm{Ma}$.

Os depósitos resultantes da "Glaciação Samburá" não são sincronizados. Não foram formados ao mesmo tempo, mas sim em um longo intervalo de tempo, no qual se desencadearam os processos tectônicos do Brasiliano (750-550 Ma). Este fato pode contribuir significantemente na dificuldade de correlacionar este tipo de depósito glaciogênico quando são envolvidas longas distâncias e/ou diferentes faixas/cinturões do Brasiliano. Também é importante observar que não é raro a presença de retrabalhamento gravitacional nos sedimentos da glaciação vendiana (Trompette 1997). Um exemplo disso seria os diamictitos da base do Grupo Vazante, da Formação Samburá e outros.

Além de algumas características de sedimentos glaciogênicos estes depósitos podem, às vezes, ser correlacionados através da presença de fosforita (Cook \& McElhinny 1979) e/ou formação ferrífera (Klein \& Beukes 1993). Em alguns depósitos podem ocorrer intercalações de minério de manganês (Walde et al. 1981).

A denominação Glaciação Samburá, para o segundo evento glacial Neoproterozóico, está sendo proposta neste trabalho para se contrapor à denominação Glaciação Jequitaí (primeiro evento glacial Neoproterozóico). Karfunkel et al. (2000) propuseram a alteração da denominação Glaciação Jequitaí para Glaciação São Francisco. Esta nova denominação da primeira glaciação Neoproterozóica é bastante oportuna e conveniente pois denota uma natureza continental sobre o Cráton do São Francisco e suas margens.

CONCLUSÕES É possível e bastante provável que um segundo evento glacial Neoproterozóico realmente tenha ocorrido em Minas Gerais e em outros estados brasileiros. Nos estados do Mato Grosso e Mato Grosso do Sul, a existência de um evento glacial Vendiano foi comprovada. Esta segunda glaciação neoproterozóica, neste trabalho denominada Glaciação Samburá, deve ter ocorrido no Vendiano e seus processos glaciais teriam transportado diamantes a longas distân- 
cias a partir do retrabalhamento de sedimentos glaciogênicos da primeira glaciação neoproterozóica (Jequitaí) e de outras fontes diamantíferas (primárias e/ou secundárias).

O estudo da Glaciação Samburá não é importante apenas para a geologia do diamante, mas também para um entendimento mais adequado da estratigrafia e da geologia regional. É fundamental que os registros de ambas as glaciações neoproterozóicas (Jequitaí e Samburá) sejam perfeitamente diferenciados.

A primeira (Jequitaí) é de natureza continental, já a segunda (Samburá), está diretamente relacionada a processos tectônicos e altitude (Evento Brasiliano). A Glaciação Samburá apresenta um registro de difícil correlação quando grandes distâncias são consideradas. Além de algumas feições glaciogênicas, a presença de fosforita e/ou formação ferrífera podem colaborar na correlação.

Agradecimentos $\mathrm{O}$ autor agradece ao profissionalismo e companheirismo do Técnico de Geologia José Tadeu Jerônimo da Silva, que o acompanhou, por mais de duas décadas, em todas as regiões brasileiras mencionadas neste trabalho como também em diversas outras. Aos revisores da RBG pelas sugestões ao manuscrito.

\section{Referências}

Alkmim F.F., Marshak, S. \& Fonseca, M.A. 2001. Assembling west Gondwana in the Neoproterozoic: clues from the São Francisco Craton region, Brazil. Geology, 29: 319-322.

Almeida, F.F.M. 1968. Evolução tectônica do centro-oeste brasileiro no Proterozóico Superior. Anais da Academia Brasileira de Ciências. 40 Suplemento: $285-295$

Alvarenga C.J.S. 1990. Pénomènes sédimentaires, strutureaux et circulation de fluides développés à la transition chaîne-craton. Exemple de la chaîne Paraguai d’âge protérozoïque supérieur, Mato Grosso, Brésil. Univ. AixMarseille III, France Thèse doct., 177p.

Alvarenga C.J.S., Moura, C.A.V., Gorayeb, P.S.S. \& Abreu F.A.M. 2000. Paraguay and Araguaia belts. In Cordani, U.G.; Milani. E.J.; Thomaz FiIho, A. \& Campos, D.A. (eds.) Tectonic Evolution of South America. $31^{\mathrm{st}}$ International Geological Congress. Rio de Janeiro, Brazil, pp.: 183-193.

Alvarenga C.J.S. \& Trompette, R. 1992. Glacial influenced turbidite sedimentation in the uppermost Proterozoic and Lower Cambrian of the Paraguay Belt (Mato Grosso, Brazil). Palaeogeography, Palaeoclimatology, Palaeoecology, 92: 85-105.

Azmy K., Veizer J., Missi A., De Oliveira, T., Dardenne, M.A. \& Sanches, A. 2000. Dolomitization and isotope stratigraphy of the Vazante Formation, São Francisco Basin, Brazil. In: $31^{\text {st }}$ International Geological Congress. Rio de Janeiro, Brazil. (2000). Abstracts, CD-ROM.

Baptista M.B., Braun, O.P.G. \& Campos D.A. 1984. Léxico Estratigráfico do Brasil. DNPM. Brasília, 222 p.

Barbosa O., Braun, O.P.G., Dyer R.C. \& Cunha C.A.B.R. 1970. Geologia da região do Triângulo Mineiro. Rio de Janeiro, DNPM/DFPM, 140 p. (Boletim 136)

Basei M.A.S., Siga Jr., O., Masquelin H.; Harara,O.M.; Reis Neto J.M. \& Preciozzi P.F. 2000. The Dom Feliciano Belt of Brazil and Uruguay and its foreland domain, the Rio de La Plata Craton: framework, tectonic evolution and correlation with similar provinces of southwestern Africa. In Cordani, U.G.; Milani. E.J.; Thomaz Filho, A. \& Campos, D.A. (eds.) Tectonic Evolution of South America. $31^{\text {st }}$ International Geological Congress. Rio de Janeiro, Brazil,pp.: 311-334.

Branco J.J.R. 1956. Conglomerado do Samburá, Minas Gerais. Anais da Academia Brasileira de Ciências, 28:295-301.

Campos J.E.G. 1996. Estratigrafia, sedimentação, evolução tectônica e geologia do diamante da porção centro-norte da Bacia Sanfranciscana.Instituto de Geociências, Universidades de Brasilia, Brasilia, Tese de doutoramento, 204 p.

Campos J.E.G. \& Gonzaga G.M. 1999. O transporte de diamantes por sistemas fluviais a longas distâncias: uma visão crítica. Revista Brasileira de Geociências., 29:113-118.

Caputo M.V. 1984. Stratigraphy, Tectonics, Paleoclimatology and Paleogeography of Northern Basins of Brazil. University of California, Santa Barbara, Tese de doutoramento, 583p.
Carvalho S.G., Brenner T.L., Zanardo A., Waring M.H. \& Feola J.L. 2000 Proterozoic phosphate deposits near Fortaleza de Minas, southwest Minas Gerais, Brazil. In: $31^{\mathrm{st}}$ International Geological Congress. Rio de Janeiro, Brazil. (2000). Abstracts, CD-ROM.

Castro P.T.A. \& Dardenne M.A. 1996. O Conglomerado Samburá (Grupo Bambuí, Neoproterozóico) e rochas sedimentares associadas no flanco leste da Serra da Pimenta, SW de Minas Gerais: um sistema de fan-delta. Geonomos, 3:35-41.

Cook P.J. \& McElhinny M.W. 1979. A reevaluation of the spatial and temporal distribution of sedimentary phosphate deposits in the light of plate tectonics. Economic Geology., 74:315-330.

Couto J.G.P. \& Bez. L. 1981. A Glaciação Jequitaí: Um guia estratigráfico para o Pré-Cambriano Superior no Brasil. Revista Brasileira de Geociências., 11:17-21.

Cukrov N.1999. A Glaciação Neoproterozóica na porção sul do Cráton do São Francisco e suas litofácies nas regiōes de Jequitaí - MG e Cristalina-GO. Instituto de Geociências, Universidades de Brasília, Brasília, Dissertaçĩo de mestrado $\mathrm{n}^{\circ} 141$.

Culver S.J. \& Williams H.R. 1978. Infracambrian glaciogenic sediments from Sierra Leone. Nature. 274:49-51.

Dardenne M.A. 2000. The Brasilia Fold Belt. In Cordani, U.G.; Milani. E.J.; Thomaz Filho, A. \& Campos, D.A (eds.) Tectonic Evolution of South America. $31^{\mathrm{s}}$ International Geological Congress. Rio de Janeiro, Brazil, рр.:231-263.

Dardenne M.A., Faria A.,Magalhães L. . \& Soares L.A. 1978. O tilito da base do Grupo Bambuí na borda ocidental do Cráton do São Francisco. Núcleo Centro Oeste. Sociedade Brasileira de Geología. p. 85-97. (Boletim7-8)

Dardenne M.A., Freitas-Silva F.H., Nogueira G.M.S. \& Souza J.C.F.1997. Depósitos de fosfotato de Rocinha e Lagamar, Minas Gerais. In. Schobbenhaus C., Queiroz, E.T. \& Coelho, C.E. S (Coords.) Principais Depósitos Minerais do Brasil. DNPM/CPRM. IVe, pp.113-122.

Dardenne M.A., Freitas-Silva F.H., Souza J.C.F. \& Campos J.E.G. 1998. Evolução tectono-sedimentar do Grupo Vazante no contexto da Faixa de Dobramentos Brasília. In: SBG/ $40^{\circ}$ Congresso Brasileiro de Geologia. Belo Horizonte Anais ..26.

Dardenne M.A. \& Schobbenhaus C. 2000. The metallogenesis of the South American Platform. In Cordani, U.G.; Milani. E.J.; Thomaz Filho, A. \& Campos, D.A. (eds.) Tectonic Evolution of South America. 31 International Geological Congress. Rio de Janeiro, Brazil, pp.: 755-850.

Dardenne M.A. \& Schobbenhaus C. 2001. Metologênese do Brasil. Editora UnBCPRM. 392p.

Derby O.1906. The Serra do Espinhaço, Brazil. The Journal of Geology, 14:374401.

Eerola T.T. 1995. From ophiolites to glaciers? Review on gelology of the Neoproterozoic-Cambrian Lavras do Sul region, southern Brazil. Geological Survey of Finland, p.5-16. Special paper20 
Evans D.A.D. 2000. An updated geochronological synthesis of the world's Neoproterozoic glaciogenic deposits. In: $31^{\text {st }}$ International Geological Congress. Rio de Janeiro, Brazil. (2000). Abstracts, CD-ROM.

Fairchild T.R. 1978. Evidências paleontológicas de uma possível idade Ediacariana ou Cambriano Inferior, para parte do Grupo Corumbá, Mato Grosso do Sul. In: SBG/ Recife, XXX Congresso Brasileiro de Geologia. Boletim Especial, p. 181.

Faria A. 1985. Geologia do Domo de Cristalina, Goiás. Revista Brasileira de Geociências, 15:231-240.

Fernandes N.H., Brenner T.L., Carvalho, S.G., Soares P.C. \& Morales N. 2000. Late Proterozoic iron and phosphatic formations in the Brasilia Colisional Belt, south Minas Gerais. Brazil. In: $31^{\mathrm{s}}$ International Geological Congress. Rio de Janeiro, Brazil. (2000). Abstracts, CD-ROM.

Freyberg B.V.1932. Ergebnisse Geologisher Forshungen in Minas Gerais (Brasilien). Stuttgart.

Frimmel H.E. \& Fölling P.G. 2000. Neoproterozoic climate changes: the perspective from southern Africa. In:31 ${ }^{\mathrm{st}}$ International Geological Congress. Rio de Janeiro, Brazil. (2000). Abstracts, CD-ROM.

Gaucher C. \& Sprechmann P. 2000. Upper Vendian skeletal fossils of the Arroyo del Soldado Group (Uruguay): New evidences on the causes of the Cambrian explosion. In: $31^{\text {st }}$ International Geological Congress. Rio de Janeiro, Brazil. (2000). Abstracts, CD-ROM.

Gonzaga G.M. \& Campos J.E.G. 2001. The importance of glacial geologic processes in the transport and concentration of diamonds: a review. In: $2^{\circ}$ Simpósio Brasileiro de Geologia do Diamante. UFMT Cuiabá-MT. Anais. Publicação Especial. 71-78.

Gonzaga G.M \& Dardenne M.A. 1991. The Jequitaí Glaciation and the dispersion of diamonds during Upper Proterozoic. In: $5^{\text {th }}$ International Kimberlite Conference. Field Guide Book. CPRM-Special Publication 3/91. P. 89-93.

Gonzaga G.M., Teixeira N.A. \& Gaspar J.C. 1994. The origin of the diamonds in the Western Minas Gerais, Brazil. Mineralium Deposita., 29:414-421.

Gonzaga G.M. \& Tompkins L.A. 1991. Geologia do Diamante. In. Schobbenhaus C., Queiroz, E.T. \& Coelho, C.E. S (Coords.) Principais Depósitos Minerais do Brasil. DNPM/CPRM. IVa, pp.53-116.

Guimarães D. 1951., DFPM/ DNPM. (Boletim 88)

Guimarães E.M., Dardenne M.A., Faria, A., Coelho C.E.S. \& Piauilino P.O.V.1986. Relação do Grupo Paranoá, Formação Jequitaí e Grupo Bambuí na região de Bezerra-GO. IN: XXXIV Congresso Brasileiro de Geologia. Goiânia. Anais 2, 853-860.

Hasui Y. \& Penalva F. 1970). O problema do diamante do Alto Paranaíba, Estado de Minas Gerais. Revista Brasileira de Geociências, 19:71-78.

Hegenberger W. 1993. Stratigraphy and sedimentology of the Late Precambrian Witvlei and Nama groups, east of Windhoek. Geological Survey of Namibia. Ministry of Mines and Energy. p. 82. ( Memoir 17)

Hoppe A., Karfunkel J. \& Noce C.M. 1984. Evidências da glaciação proterozóica no centro-sul do Estado de Minas Gerais. Ciências da Terra., 9:16.

Karfunkel J., Dupont H., Hoppe A., Schobbenhaus C., Noce C.M. \& Peregovich B. 2000. The Neoproterozoic São Francisco Glaciation in central-eastern Brazil. In: XVII Simposio sobre la Geología de Latinoamérica. Universität Stuttgart.

Karfunkel J., Martins M.S., Schols R. \& McCandless T. 2001. Diamonds from the Macaúbas River Basin (MG): Characteristics and possible source. In: $3^{\text {rd }}$ Brazilian Symposium on diamond Geology \& $1^{\text {st }}$ South American Symposium on Diamond Geology. Brasília, DF-Brazil. Abstracts, p.23

Karfunkel J., \& Noce C.M. 1983. Desenvolvimento faciológico do PréCambriano Superior da região de Carandaí - São João Del Rei, Minas Gerais. In: II Simpósio de Geologia de Minas Gerais. Belo Horizonte, Anais, 16-29.

Karfunkel J., Noce C.M. \& Hoppe A. 1983. A Formação Carandaí no centro-sul do Estado de Minas Gerais, gênese, ambiente de deposição e correlações. In: II Simpósio de Geologia de Minas Gerais. Belo Horizonte, Atas, 30-37.

Kaufman A. J. 1998. Neoproterozoic chemostratigraphy: key events in earth history ordered by detailed intra-and inter-basinal correlation. In: SBG/ XL Congresso Brasileiro de Geologia, Belo Horizonte, Anais, 2.

Klein C. \& Beukes N.J. 1993. Sedimentology and geochemistry of the glaciogenic
Late Proterozoic Raptian Iron-Formation in Canada. Economic Geology., 88. $542-565$.

Leonardos O.H. 1940. Tilito metamórfico de Carandaí, Minas Gerais. Anais da Academia Brasileira de Ciências., 12:243-259.

Moares L.I. \& Guimarães D. 1930. Geologia da região diamantífera do norte de Minas Gerais. Rio de Janeiro. DNPM, p. 115-138. (Boletim 19)

Pedrosa-Soares A.C., Cordani U.G. \& Nutman A. 2000. Constraining the age of Neoproterozoic Glaciation in Eastern Brazil: First U-Pb (SHRIMP) data of detrital zircons. Revista Brasileira de Geociências., 30:58-61.

Pereira L.F. 1992. Relações tectono-estratigráficas entre as unidades Canastra e Ibiá na região de Coromandel, MG. Instituto de Geociências, Universidades de Brasília, Brasília, Dissertação de mestrado, 73p.

Pimentel M.M., Fuck R.A. \& Botelho N.F. 1999. Granites and the geodynamic history of the Neoproterozoic Brasília Belt, central Brazil: A review. Lithos., 46:463-483.

Reis E. 1959. Os grandes diamantes brasileiros. Rio de Janeiro. DNPM/DGM. p. 6.5. (Boletim $n^{\circ}$ 191)

Rocha-Campos A.C. \& Hasui Y. 1981. Late Precambrian Salobro Formation. In: Hambrey, M. J. \& Harland, W. B. (eds), Earth Pre-Pleistocene Glacial Record. Cambridge Univ. Press, pp.: 928-930.

Rosa C.L.M. 2000. Colonization of Vendian marginal marine environment: Ichnofauna from the Santa Barbara Alogroup, southern Brazil. In: $31^{\text {st }}$ International Geological Congress. Rio de Janeiro, Brazil. (2000). Abstracts, CD-ROM.

Rouffaer E.J.H.F. 1988. On the origin of alluvial diamonds along the Atlantic coast of South Africa and South West Africa. Leuven. Belgium, Phd. Thesis, $170 \mathrm{p}$.

Schermerhorn L.J.G. 1983. Late Proterozoic glaciation in the light of $\mathrm{CO}_{2}$ depletion in the atmosphere in: Proterozoic Geology: Selected papers from an International Proterozoic symposium. Edited by Medaris Jr., L. G.; Byers, C. W., Mickelson D. M. \& Shanks W. C. The Geological Society of America. P.309-315. (Memoir 161)

Schobbenhaus C., Campos D.A., Derze G.R. \& Asmus H.E. (Coordenadores) 1981. Mapa geológico do Brasil e da área oceânica adjacente incluindo depósitos minerais. Escala I:2.500.000. Brasília.DNPM.

Seer H.J., Moraes L.C. \& Fogaça A.C.C. 1987. Faciologia e estruturação tectônica dos metassedimentos com diamictitos e jaspelitos da região de Lagoa Formosa-MG (Gr. Bambuí?). In: $4^{\circ}$ Simpósio de Geologia de Minas Gerais, Anais, 199-213.

Silva Filho M.A. 1974, "Projeto sul da Bahia”. Relatório final. Convênio. Salvador. DNPM/CPRM. V. 15. (Relatório. Inédito).

Souza J.C.F. 1997. Litoestratigrafia e sedimentologia da Formação Vazante na região de Coromandel, MG. Instituto de Geociências, Universidades de Brasilia, Brasilia, Tese de mestrado, 75p.

Torquato J.R. \& Cordani U.G. 1981. Brazil-Africa Geologiacal Links. EarthScience, Reviews.17:155-176.

Trompette R. 1994. Geology of Western Gondwana (2000-500 Ma). Pan-AfricanBrasiliano aggregation of South America and Africa. A. A. Balkema. 350p.

Trompette R. 1997. Neoproterozoic ( $600 \mathrm{Ma})$ aggregation of Western Gondwana: a tentative scenario. Precambrian Research., 82:101-112.

Trouw R., Heilbron M., Ribeiro A., Paciullo F., Valeriano C.M., Almeida J.C.H., Tupinambá M. \& Andreis R.R.2000. The central segment of the Ribeira Belt. In Cordani, U.G.; Milani. E.J.; Thomaz Filho, A. \& Campos, D.A

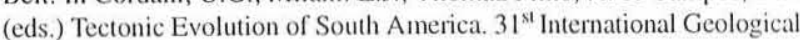
Congress. Rio de Janeiro, Brazil, pp.:287-310.

Valeriano C.M. 1999. A Faixa Brasília meridional com ênfase no segmento da Represa de Furnas: modelos de evolução tectônica. Universidade Estadual do Rio de Janeiro, Rio de Janeiro, Tese de Livre Docência, 89p.

Van Wyk J.P. \& Pienaar L.F. 1986. Diamondiferous gravels of the lower Orange River, Namaqualand. In by Anhaeusser, C. R. \& Maske, S. (eds.) Mineral deposits of southern Africa, II. The Geological Society of South Africa, pp. $2309-2321$

Villeneuve M. 1987. Geodynamic evolution of the Mauritanide, Bassaride, and Rokelide orogens (West Africa). Precambrian Research., 37:19-28.

Walde D.H.G., Gierth E. \& Leonardos O.H. 1981. Stratigraphy and mineralogy 
of the manganese ores of Urucum, Mato Grosso, Brazil. Geol. Rundschau., 70:1077-1085

Walde D.H.G., Leonardos O.H., Hahn G. \& Pflug H.D. 1982. The first PreCambrian megafossils from South America, Corumbelia Wemeri. Anais da Academia Brasileira de Ciências., 54:461.
Manuscrito A-1311 Recebido em 01 de dezembro de 200 Revisão dos autores em 20 de dezembro de 2001 Revisão aceita $\mathrm{em} 21$ de dezembro de 2001 